\title{
Systematic analysis of mRNA expression profiles in NSCLC cell lines to screen metastasis-related genes
}

\author{
YING LIU*, LEI LIU*, TAO YU, HE-CHUN LIN, DANDAN CHU, \\ WEI DENG, MING-XIA YAN, JING LI and MING YAO
}

State Key Laboratory of Oncogenes and Related Genes, Shanghai Cancer Institute, Renji Hospital, Shanghai Jiao Tong University School of Medicine, Shanghai 200032, P.R. China

Received September 14, 2015; Accepted September 29, 2016

DOI: $10.3892 / \mathrm{mmr} .2016 .5911$

\begin{abstract}
Lung cancer is the most prevalent cancer in humans and has the lowest survival outcomes due to its high metastatic potential. The aim of the present study was to screen for metastasis-related genes (MRGs) by investigating the differential expression genes (DEGs) identified by the mRNA expression profiles in SPC-A-1sci (highly metastatic) and SPC-A-1 (parental) cells. DEGs were screened using Genespring software. Gene Ontology and pathway enrichment analyses of these DEGs were performed. Interaction networks between the proteins encoded by the DEGs were identified using the database BioGRID and were visualized by Cytoscape. Modular analysis of the protein-protein interaction network was performed in CFinder. Among these DEGs, the expression levels of 18 genes were examined in SPC-A-1sci and SPC-A-1 cell lines with reverse transcription-quantitative polymerase chain reaction, and 10 of the 18 genes were assessed by western blotting to validate the results of the microarray. Furthermore, the role of metallothionein 1X (MT1X) in non-small cell lung cancer was explored in functional assays and 72 pairs of clinical samples in vitro. Finally, 4,838 DEGs were screened, including 798 upregulated and 4,040 downregulated genes. The significantly enriched functions included gene expression, cytosol and poly-(A) RNA binding, and the most enriched pathway was biosynthesis of antibiotics. Furthermore, MT1X was revealed to promote the migration and invasion ability in SPC-A-1sci and PC-9 lung cancer cell lines. Therefore, MT1X was identified as a candidate MRG through systematic
\end{abstract}

Correspondence to: Dr Ming Yao or Ms. Jing Li, State Key Laboratory of Oncogenes and Related Genes, Shanghai Cancer Institute, Renji Hospital, Shanghai Jiao Tong University School of Medicine, 25 Lane 2200 Xietu Road, Shanghai 200032, P.R. China

E-mail: myao@shsci.org

E-mail: shuixingyixin@126.com

*Contributed equally

Key words: mRNA expression profile, non-small cell lung cancer, metastasis, gene, prognosis analysis in the present microarray, which was demonstrated to offer potential reference value in screening MRGs.

\section{Introduction}

Lung cancer is reported to be the leading cause of cancer-associated mortality among males in developed and developing countries, and its mortality is the leading cause among females in developed countries $(1,2)$. Non-small cell lung cancer (NSCLC) occupies the majority of the lung cancer cases and exhibits a high mortality rate, and a low 5-year survival rate (3). A high metastatic potential of NSCLC generates the lowest survival outcome (4). The complex process of tumorigenesis and metastasis is due to a variety of factors, which remain to be elucidated. Therefore, exploring the molecular mechanisms of NSCLC metastasis is required for more effective clinical therapies.

Numerous studies have performed high-throughput microarray technology and analysis methods for investigating the molecular mechanisms in human diseases. For example, the study by Sun et al (5) performed an integrative microarray approach to analyze the genome-wide mRNA expression in osteosarcoma cell lines and identified 8 'hub' genes that appeared to be involved in osteosarcoma (5). Huang et al (6) identified epithelial-mesenchymal transition-associated prognostic biomarkers that predicted the distant metastasis of lung cancer using DNA microarray and survival data (6). The widespread use of high-throughput technologies allows for the simultaneous and convenient comprehensive examination of the global gene expression. Application of these technologies can identify genes that may be used as novel molecular targets for clinical treatment. The bioinformatics analysis revealed more information with regards to the significant functions, pathways, conceivable connections and signaling of these differentially expressed genes (DEGs). The interaction among DEGs, particularly the functional modules in the interaction network, also remain to be elucidated for the molecular mechanisms of metastasis. A previous study established a highly metastatic lung cancer cell subline (SPC-A-1sci) from a weakly metastatic cell line (SPC-A-1) through in vivo selection in NOD/SCID mice (7). This pair of cell lines provided an appropriate model for exploring the mechanisms of NSCLC metastasis. Therefore, microarray analysis of this pair of cell 
lines was performed to identify the metastasis-related genes (MRGs) from the mRNA expression profiles with comprehensive array analysis and in vitro experiments.

Metallothionein 1X (MT1X) is involved in mineral absorption and organism-specific biosystems. The $\mathrm{T}([20])$ repeat in the 3'-untranslated region of the MT1X gene has been reported to be a sensitive and specific marker for detecting microsatellite instability in colorectal cancer (8). A development of cisplatin resistance was confirmed following knockdown of MT1X (9). The present study observed that a knockdown of MT1X decreased the metastatic ability of the NSCLC cell lines through a series of in vitro experiments. This finding confirmed that the present microarray offers valuable information regarding the metastatic mechanisms of NSCLC.

\section{Materials and methods}

Cell lines and cell culture. The SPC-A-1 human lung cancer cell line was originally isolated from the surgical specimens of a Chinese patient with advanced lung adenocarcinoma at the Shanghai Chest Hospital and Cellular Institute of Chinese Academy of Science (Shanghai, China). The highly metastatic lung cancer cell line, SPC-A-1sci, was obtained from the Cellular Institute of Chinese Academy of Science (Shanghai, China) and was established by Professor Ming Yao (Shanghai Jiaotong University, The Shanghai Cancer Institute, Shanghai, China) from the weakly metastatic cell line (SPC-A-1) through selection in NOD/SCID mouse models (7). A549, H1299, PC-9, LC-21, H358, H292, SPC-A-1 and SPC-A-1sci NSCLC cell lines (all from the American Type Culture Collection, Manassas, VA, USA) were cultured in Dulbecco's modified Eagle's medium (Thermo Fisher Scientific, Inc., Waltham, MA, USA), supplemented with $10 \%$ fetal bovine serum (South America origin; Biowest USA, Riverside, MO, USA), $100 \mathrm{U} / \mathrm{ml}$ penicillin (Sigma-Aldrich; Merck Millipore, Darmstadt, Germany) and $100 \mu \mathrm{g} / \mathrm{ml}$ streptomycin (Sigma-Aldrich; Merck Millipore) in a humidified incubator at $37^{\circ} \mathrm{C}$ with $5 \% \mathrm{CO}_{2}$.

Microarray data analysis. The total RNA from each cell line was harvested using the RNeasy Mini kit (Qiagen $\mathrm{GmbH}$, Hilden, Germany), according to the manufacturer's instructions. In total, 6 specimens, including three replicates of SPC-A-1sci and three replicates of SPC-A-1 RNA specimens, were sent to Shanghai OE-Biotech Co., Ltd. (Shanghai, China) and were processed according to the Agilent Technologies, Inc. (Santa Clara, CA, USA) technical instructions. Feature Extraction software (version 10.7.1.1; Agilent Technologies, Inc.) was used to analyze the array images to obtain the raw data. Genespring software (version 12.5; Agilent Technologies, Inc.) was used to finish the basic analysis with the raw data that was normalized with the quantile algorithm.

DEGs screening. The probes with $\geq 1$ of 2 conditions which have flags in ' $\mathrm{P}$ ' were selected for further data analysis. DEGs were subsequently identified through fold change, and the $\mathrm{P}$-value calculated with the $t$-test. The threshold set for up and downregulated genes was a fold change $\geq 2.0$ and $\mathrm{P} \leq 0.05$.

Functional analysis and pathway enrichment analysis. Gene-annotation enrichment analyses were applied to determine the roles of these DEGs. Gene Ontology (GO; www.geneontology.org/) provides extensive details, which consists of biological process, cellular components and molecular functions (10). The Kyoto Encyclopedia of Genes and Genomes (KEGG; www.genome.ad.jp/kegg/) is a comprehensive database resource and forms a high-throughput data-mining environment (11).

Protein-protein interaction (PPI) network and module construction. Interaction networks between the proteins encoded by the DEGs were identified using the database, BioGRID 3.4 (thebiogrid.org/), and were visualized by Cytoscape 3.2 (cytoscape.org/). Modular analysis of the PPI network was performed in ClusterONE (/www.paccanarolab. Org.sci-hub.org/clusterone/). The cut-off criterion was false discovery rate $<0.01$ and the clique value $(\mathrm{k})$ was 6 .

Small interfering RNA (siRNA) transfection. siRNA oligonucleotides were synthesized by Guangzhou RiboBio Co., Ltd. (Guangdong, China). Lipofectamine 2000 reagent (Invitrogen; Thermo Fisher Scientific, Inc.) was used to deliver siRNAs into the cells, according to the manufacturer's instructions. Briefly, $3 \times 10^{5}$ cells/well were plated into 6 -well plates and cultured overnight to achieve 50-70\% confluence, and were subsequently washed with PBS. A mixture of Lipofectamine 2000 reagent and siRNA was added to the cells to final concentrations of $5 \mathrm{ml} /$ well and $2.5 \mathrm{mg} /$ well, respectively, followed by incubation at $37^{\circ} \mathrm{C}$ for $48 \mathrm{~h}$. The transfection efficiency was determined by reverse transcription-polymerase chain reaction (RT-PCR).

Preparation of the protein extracts. All procedures were performed on ice. Proteins from the cultured SPC-A-1sci and SPC-A-1 cell lines were harvested from $8-\mathrm{cm}$ culture plates, rinsed with cold PBS three times, scraped using a cell scraper, and were subsequently transferred into $1.5 \mathrm{ml}$ tubes and lysed with $200 \mathrm{ml}$ ice-cold lysis buffer per tube. After a $40 \mathrm{~min}$ incubation on ice, the lysates were centrifuged at $12,000 \mathrm{x} g$ for $10 \mathrm{~min}$ at $4^{\circ} \mathrm{C}$. The supernatants were collected and stored at $-20^{\circ} \mathrm{C}$. Protein concentrations were measured with a bicinchoninic acid protein assay kit (Thermo Fisher Scientific, Inc.).

Western blot analysis. The protein samples $(20 \mu \mathrm{g})$ were run on a $10 \%$ SDS-PAGE gel and were transferred onto nitrocellulose (NC) membranes. The NC membranes with protein were blocked in $5 \%$ fat-free powdered milk at room temperature for $2 \mathrm{~h}$. The primary antibodies used areas follows: Primary rabbit polyclonal anti-A-kinase anchoring protein 12 (AKAP12; 1:200 dilution; cat. no. 25199-1-AP; ProteinTech Group, Inc., Chicago, IL, USA), rabbit polyclonal anti-aldehyde dehydrogenase 7 family member A1 (ALDH7A1; 1:5,000 dilution; cat. no. 2070-S; Epitomics, Burlingame, CA, USA), rabbit polyclonal anti-caveolin 1 (CAV1; cat. no. 21112-1; 1:500 dilution; Signalway Antibody LLC, College Park, MD, USA), rabbit polyclonal anti-erb-b2 receptor tyrosine kinase 2 (Erbb2; 1:1,000 dilution; cat. no. 2165s; Cell Signaling Technology, Inc., Danvers, MA, USA), rabbit polyclonal anti-growth arrest and DNA damage inducible $\alpha$ (GADD45 $\alpha$; 1:200 dilution; cat. no. sc-797; Santa Cruz Biotechnology, Inc., Dallas, TX, USA), goat polyclonal anti-keratin 18 (Krt18; 1:200 dilution; cat. no. 35624; Signalway Antibody 
LLC), goat polyclonal anti-myosin heavy chain 9 (MYH9; 1:500 dilution; cat. no. c2910; Santa Cruz Biotechnology, Inc.), goat polyclonal anti-proliferating cell nuclear antigen (PCNA; 1:100 dilution; cat. no. AF0239; Affinity Bioscience, Cincinnati, $\mathrm{OH}$, USA), rabbit polyclonal anti-carbamoyl-phosphate synthase 1 (CPS1; 1:200 dilution; cat. no. AP16053a; Abgent, Inc., San Diego, CA, USA), rabbit polyclonal anti-phosphoglycerate dehydrogenase (PHGDH; 1:300 dilution; cat. no. AP2936c; Abgent, Inc.) and anti- $\beta$-actin (1:5,000; cat. no. ab14128; Abcam, Cambridge, MA, USA). The secondary antibodies were anti-goat-horseradish peroxidase (HRP) (cat. no. A5420) and anti-rabbit-HRP (RABHRP1) (1:200; Sigma-Aldrich; Merck Millipore). $\beta$-actin, was used as a normalization for all the protein samples. Primary antibodies were added and incubated for $1 \mathrm{~h}$ at room temperature and subsequently overnight at $4^{\circ} \mathrm{C}$. Following three washes in Tris-buffered saline and Tween-20 (TBST; $10 \mathrm{~min} /$ wash), the corresponding secondary antibody solution was added and the membranes were incubated for $1 \mathrm{~h}$ at room temperature. Subsequent to washing three times in TBST, immunolabeling was visualized by electrochemiluminescence (EMD Millipore, Billerica, MA, USA) and the chemiluminescence was developed using film.

$R T$-quantitative PCR (RT-qPCR) analysis. RNA samples were harvested using TRIzol reagent (Invitrogen; Thermo Fisher Scientific, Inc.). The RT reagents were used for the RT process at $37^{\circ} \mathrm{C}$ for $15 \mathrm{~min}$ and $85^{\circ} \mathrm{C}$ for $5 \mathrm{sec}$ and were obtained from Takara Biotechnology Co., Ltd. (Dalian, China). Primary data were analyzed on a 7300 Real-Time PCR system with SDS RQ Study software (Applied Biosystems; Thermo Fisher Scientific, Inc.), according to the manufacturer's instructions. The cycling conditions were as follows: Initial denaturation at $95^{\circ} \mathrm{C}$ for $10 \mathrm{sec}, 40$ cyles of $95^{\circ} \mathrm{C}$ for $5 \mathrm{sec}$ and $60^{\circ} \mathrm{C}$ for $31 \mathrm{sec}$. cDNA templates were combined with SYBR-Green premix, containing ROX (Takara Biotechnology Co., Ltd.), to perform the RT-qPCR reactions. $\beta$-actin was used as the endogenous control for quantifying the mRNA levels. All the primers were provided by Shanghai Sunny Biotechnology Co., Ltd. (Shanghai, China; Table I). The reactions were performed in triplicate and the $2^{-\Delta \Delta C q}$ method (12) was used for quantification.

Migration and invasion assays. Cell migration and invasion assays were performed with 6.5-mm Transwell chambers (8-mm pore size; Corning Incorporated, Corning, NY, USA). The cells were seeded at 100,000 cells per well into Transwell chambers and Matrigel-coated Transwell chambers for the migration and invasion assays, respectively. After $16 \mathrm{~h}$ incubation for the two assays, the cells that remained on the upper chamber were removed with a cotton swab, and the entire chamber was fixed with methyl alcohol and stained with crystal violet. Migrated cells were counted using a CKX41 microscope (Olympus Corporation, Tokyo, Japan) at x400 magnification and a DP20 Imaging system (Olympus Corporation). Images from three random fields from three replicate wells were captured and the number of cells that had migrated and invaded were counted.

Statistical analysis. The results are presented as the mean \pm standard deviation. Comparisons of the quantitative data were analyzed by the Student's t-test between two groups (two-tailed). Differences between more than one group were analyzed using a one-way analysis of variance with Bonferroni post-hoc test. $\mathrm{P}<0.05$ was considered to indicate a statistically significant difference. Analyses were performed with SPSS 19.0 for Windows (IBM SPSS, Armonk, NY, USA).

\section{Results}

DEG analysis. In total, 4,838 DEGs were screened, including 798 upregulated genes and 4,040 downregulated genes (fold change $\geq 2.0$ and $\mathrm{P} \leq 0.05$ ). There were more downregulated compared with upregulated genes.

Functional analysis and pathway enrichment analysis. The 10 most enriched GO functions for the DEGs are listed in Tables II-IV. For the upregulated genes, the enriched functions in the biological process category included gene expression $(\mathrm{P}=1.46 \mathrm{E}-28)$, translation $(\mathrm{P}=1.46 \mathrm{E}-16)$, viral process $(\mathrm{P}=5.94 \mathrm{E}-16)$ and the cellular protein metabolic process $(\mathrm{P}=52.06 \mathrm{E}-13)$. The enriched cellular component categories were cytosol $(\mathrm{P}=1.96 \mathrm{E}-31)$, membrane $(\mathrm{P}=52.79 \mathrm{E}-29)$, nucleoplasm $(\mathrm{P}=51.56 \mathrm{E}-25)$ and mitochondrion $(\mathrm{P}=5.02 \mathrm{E}-23)$. The enriched molecular function categories were poly-(A) RNA binding $(\mathrm{P}=1.46 \mathrm{E}-28)$, protein binding $(\mathrm{P}=1.46 \mathrm{E}-16)$, structural constituent of the ribosome $(\mathrm{P}=5.94 \mathrm{E}-16)$ and $\mathrm{RNA}$ binding $(\mathrm{P}=2.06 \mathrm{E}-13)$. The 10 most enriched KEGG pathways for the DEGs are listed in Table V. DEGs were predominantly enriched in the biosynthesis of antibiotics $(\mathrm{P}=5.81 \mathrm{E}-08)$, carbon metabolism $(\mathrm{P}=5.20 \mathrm{E}-07)$, spliceosome $(\mathrm{P}=5.81 \mathrm{E}-07)$ and ribosome $(\mathrm{P}=7.39 \mathrm{E}-07)$.

PPI network and module construction. The PPI network of the DEGs was revealed to have 426 nodes and 1,156 interactions (Fig. 1). Cluster analysis was performed with ClusterONE. A total of 56 modules were obtained from the PPI network, of which the top two modules (modules A and B) are presented in Fig. 2. The enriched pathways for the DEGs in the two modules are listed in Table VI. Module A had 31 nodes and 36 interactions. The four most enriched KEGG pathways for DEGs in module A were biosynthesis of antibiotics $(\mathrm{P}=5.81 \mathrm{E}-08)$, protein processing in the endoplasmic reticulum $(\mathrm{P}=2.66 \mathrm{E}-05)$, $\mathrm{RNA}$ transport $(\mathrm{P}=0.00093377)$ and pyrimidine metabolism $(\mathrm{P}=0.034919775)$. The module density was 0.103 , quality was 0.353 and $\mathrm{P}=1.151 \mathrm{E}-7$. Module A predominantly consisted of downregulated genes and its 'hub' gene was nuclear RNA export factor 1 (NXF1) with a degree of 107. In this module, 11 genes were identified to participate in the mRNA transport pathway, including NXF1, nucleoporin (NUP) 88, NUP188, NUP50, karyopherin subunit $\beta 1$ (KPNB1), exportin (XPO) 1, NUP62, NUP93, RAN member RAS oncogene family, snurportin 1 and XPO5. NXF1 transports mRNA by bulk export pathways (13). The interactome network analysis in the expression profiles of the precursor-B-cell acute lymphoblastic leukemia cell lines in a previous study predicted that bulk dysregulation of NXF1 predicted poor prognosis for acute lymphoblastic leukemia, and numerous mRNA export factors, including NXF1 and NUP88, are deemed to be potential therapeutic targets in cancer (14).

Module B had 6 nodes and 15 interactions. In this module, DEGs were predominantly enriched in RNA transport 


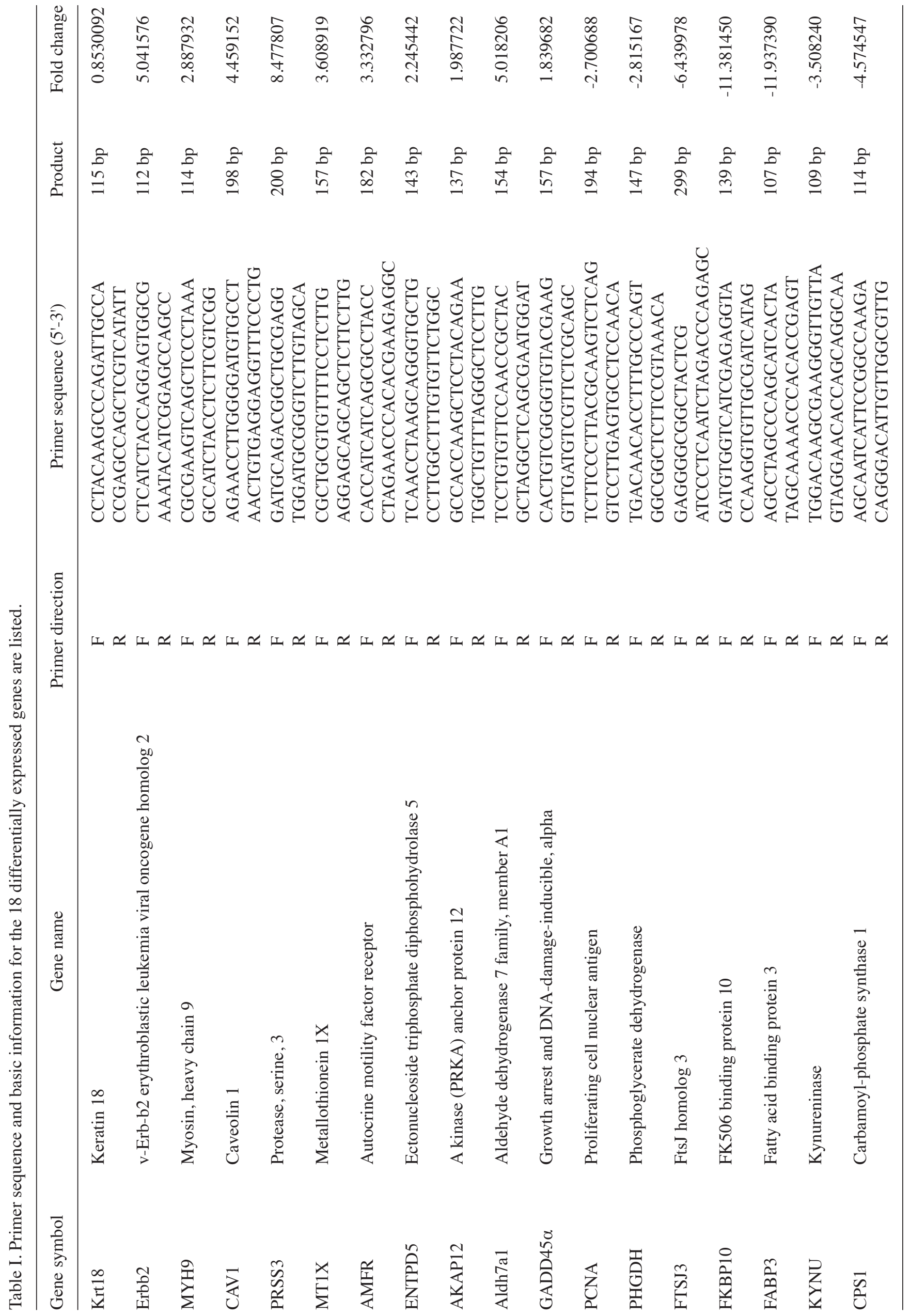


Table II. Top ten significantly enriched BP terms in non-small cell lung cancer cell lines.

\begin{tabular}{|c|c|c|c|c|}
\hline Term & Category & Description & Count & P-value \\
\hline GO:0010467 & $\mathrm{BP}$ & Gene expression & 364 & $1.46 \mathrm{E}-28$ \\
\hline GO:0006412 & $\mathrm{BP}$ & Translation & 127 & $1.46 \mathrm{E}-16$ \\
\hline GO:0016032 & $\mathrm{BP}$ & Viral process & 228 & $5.94 \mathrm{E}-16$ \\
\hline GO:0044267 & $\mathrm{BP}$ & Cellular protein metabolic process & 239 & $2.06 \mathrm{E}-13$ \\
\hline GO:0000278 & $\mathrm{BP}$ & Mitotic cell cycle & 172 & $2.55 \mathrm{E}-12$ \\
\hline GO:0006614 & $\mathrm{BP}$ & SRP-dependent cotranslational protein targeting to membrane & 65 & $2.31 \mathrm{E}-10$ \\
\hline GO:0044281 & $\mathrm{BP}$ & Small molecule metabolic process & 430 & $2.81 \mathrm{E}-10$ \\
\hline GO:0019058 & $\mathrm{BP}$ & Viral life cycle & 77 & $4.81 \mathrm{E}-10$ \\
\hline GO:0006413 & $\mathrm{BP}$ & Translational initiation & 74 & $5.82 \mathrm{E}-10$ \\
\hline GO:0008380 & $\mathrm{BP}$ & RNA splicing & 108 & $8.55 \mathrm{E}-10$ \\
\hline
\end{tabular}

$\mathrm{GO}$, gene ontology; BP, biological process.

Table III. Top ten significantly enriched CC terms in non-small cell lung cancer cell lines.

\begin{tabular}{|c|c|c|c|c|}
\hline Term & Category & Description & Count & P-value \\
\hline GO:0010467 & $\mathrm{CC}$ & Cytosol & 959 & $1.96 \mathrm{E}-31$ \\
\hline GO:0006412 & $\mathrm{CC}$ & Membrane & 657 & $2.79 \mathrm{E}-29$ \\
\hline GO:0016032 & $\mathrm{CC}$ & Nucleoplasm & 815 & $1.56 \mathrm{E}-25$ \\
\hline GO:0044267 & $\mathrm{CC}$ & Mitochondrion & 449 & $5.02 \mathrm{E}-23$ \\
\hline GO:0000278 & $\mathrm{CC}$ & Extracellular exosome & 835 & 8.88E-23 \\
\hline GO:0006614 & $\mathrm{CC}$ & Cytoplasm & 1308 & $3.17 \mathrm{E}-22$ \\
\hline GO:0044281 & $\mathrm{CC}$ & Nucleolus & 310 & $1.19 \mathrm{E}-16$ \\
\hline GO:0019058 & $\mathrm{CC}$ & Focal adhesion & 163 & $1.58 \mathrm{E}-11$ \\
\hline GO:0006413 & $\mathrm{CC}$ & Nucleus & 1247 & $1.00 \mathrm{E}-10$ \\
\hline GO:0008380 & $\mathrm{CC}$ & Mitochondrial inner membrane & 166 & $1.01 \mathrm{E}-10$ \\
\hline
\end{tabular}

GO, gene ontology; CC, cellular compartment.

Table IV. Top ten significantly enriched MF terms in non-small cell lung cancer cell lines.

\begin{tabular}{|c|c|c|c|c|}
\hline Term & Category & Description & Count & P-value \\
\hline GO:0010467 & MF & Poly (A) RNA binding & 364 & $1.46 \mathrm{E}-28$ \\
\hline GO:0006412 & MF & Protein binding & 127 & $1.46 \mathrm{E}-16$ \\
\hline GO:0016032 & MF & Structural constituent of ribosome & 228 & $5.94 \mathrm{E}-16$ \\
\hline GO:0044267 & MF & RNA binding & 239 & $2.06 \mathrm{E}-13$ \\
\hline GO:0000278 & MF & Identical protein binding & 172 & $2.55 \mathrm{E}-12$ \\
\hline GO:0006614 & MF & Enzyme binding & 65 & $2.31 \mathrm{E}-10$ \\
\hline GO:0044281 & MF & Protein domain specific binding & 430 & $2.81 \mathrm{E}-10$ \\
\hline GO:0019058 & $\mathrm{MF}$ & ATP binding & 77 & $4.81 \mathrm{E}-10$ \\
\hline GO:0006413 & MF & Unfolded protein binding & 74 & $5.82 \mathrm{E}-10$ \\
\hline GO:0008380 & MF & Transcription factor binding & 108 & $8.55 \mathrm{E}-10$ \\
\hline
\end{tabular}

GO, gene ontology; MF, molecular function.

( $\mathrm{P}=1.63 \mathrm{E}-05)$, viral myocarditis $(\mathrm{P}=0.07922721)$, proteoglycans in cancer $(\mathrm{P}=0.134513214)$ and the mechanistic target of rapamycin (mTOR) signaling pathway $(\mathrm{P}=0.255698228)$. The module density was 0.764 , quality was 0.689 and $\mathrm{P}=5.248 \mathrm{E}-7$. Module B consisted of 11 downregulated genes and its 'hub' genes were eukaryotic translation initiation factor (EIF) 3A, 
Table V. Top ten significantly enriched Kyoto Encyclopedia of Genes and Genomes pathway in non-small cell lung cancer cell lines.

\begin{tabular}{lrll}
\hline Pathway & Count & \multicolumn{1}{c}{ Term } & P-value \\
\hline Path:hsa01130 & 101 & Biosynthesis of antibiotics & $5.81 \mathrm{E}-08$ \\
Path:hsa01200 & 62 & Carbon metabolism & $5.20 \mathrm{E}-07$ \\
Path:hsa03040 & 69 & Spliceosome & $5.81 \mathrm{E}-07$ \\
Path:hsa03010 & 70 & Ribosome & $7.39 \mathrm{E}-07$ \\
Path:hsa03013 & 76 & RNA transport & $1.63 \mathrm{E}-05$ \\
Path:hsa04141 & 74 & Protein processing in endoplasmic reticulum & $2.66 \mathrm{E}-05$ \\
Path:hsa05012 & 63 & Parkinson's disease & $9.04 \mathrm{E}-05$ \\
Path:hsa01230 & 39 & Biosynthesis of amino acids & 0.000125444 \\
Path:hsa00190 & 59 & Oxidative phosphorylation & 0.00012915 \\
Path:hsa04932 & 64 & Non-alcoholic fatty liver disease & 0.000189471 \\
\hline
\end{tabular}

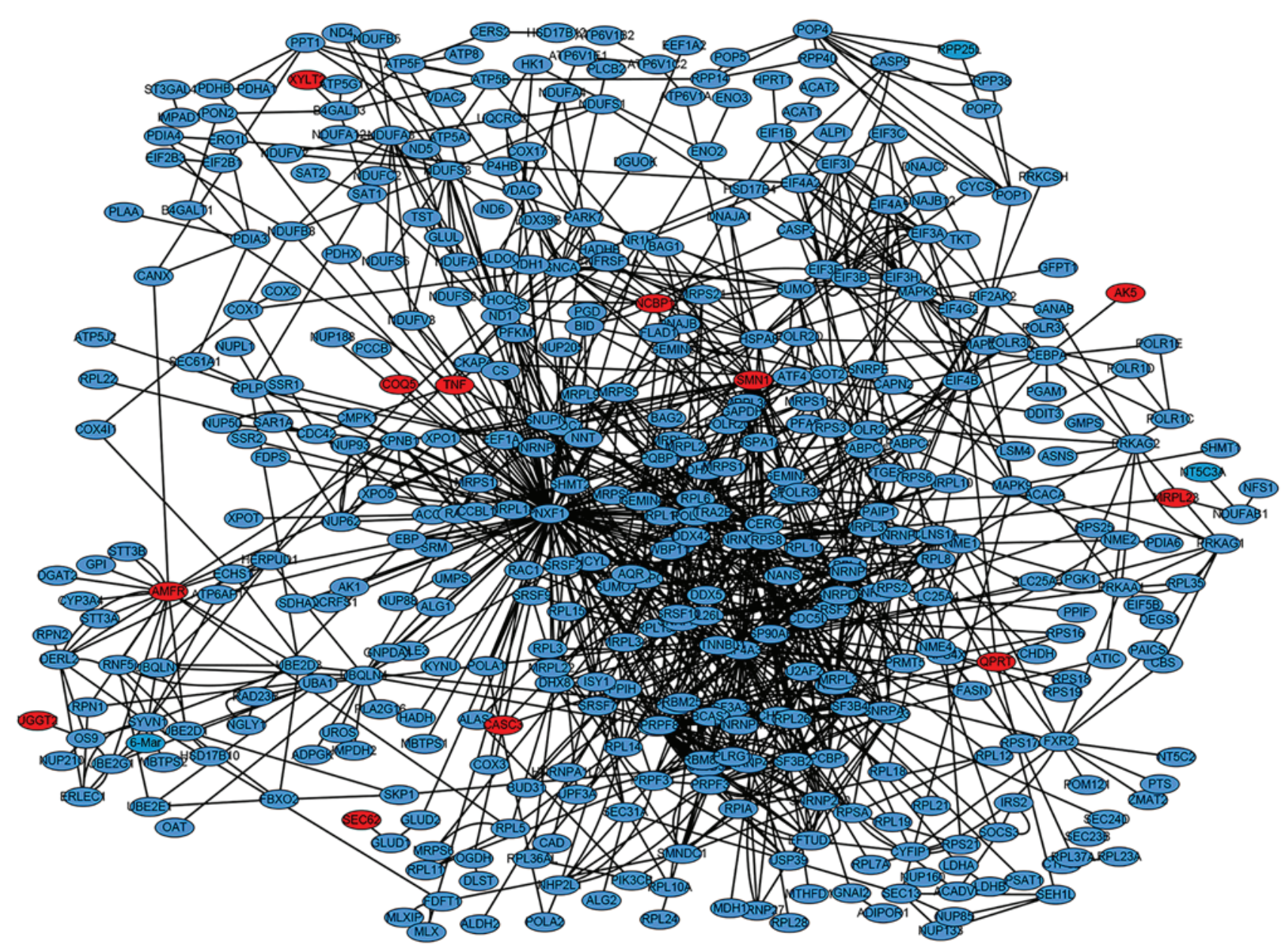

Figure 1. Protein-protein interaction network of the DEGs. The red and blue circles represent for the upregulated and downregulated genes, respectively.

EIF3F and EIF3B, with the same degree of 15. EIF3A has been previously reported to improve cisplatin sensitivity in ovarian cancer, and its overexpression in urinary bladder cancer and NSCLC can provide a prognostic value (15-17). EIF3F is downregulated in several cancer types, particularly in melanoma and pancreatic cancer cells, and has a tumor suppressive effect $(18,19)$. EIF3B is reported to inhibit migration in NSCLC (20).
RT-qPCR and western blotting validation of the expression of selected DEGs. Notably, among the DEGs, insulin-like growth factor-II mRNA binding protein 3, lipocalin2, colony stimulating factor 1, annexin A2 and suppressor of cytokine signaling 3 were identified as biomarkers for tumors (21-26). In addition, epidermal growth factor receptor (EGFR), S100 calcium binding protein (S100) P, S100A2, Erbb2, metastasis associated 1 (MTA1) and mTOR are widely recognized to be involved 
Table VI. Enriched Kyoto Encyclopedia of Genes and Genomes pathways for differentially expressed genes in modules A-B.

\begin{tabular}{|c|c|c|c|c|}
\hline Module & Pathway & Numbers of genes & Examples of genes & $\mathrm{P}$-value \\
\hline \multirow[t]{4}{*}{ A } & Biosynthesis of antibiotics & 4 & PCCB, SDHA, HADH, IDH1 & $5.81 \mathrm{E}-08$ \\
\hline & $\begin{array}{l}\text { Protein processing in endoplasmic } \\
\text { reticulum }\end{array}$ & 2 & IDH1, SDHA & $2.66 \mathrm{E}-05$ \\
\hline & RNA transport & 11 & $\begin{array}{c}\text { NXF1, NUP88, NUP188, } \\
\text { NUP50 KPNB1, XPO1, NUP62, } \\
\text { NUP93, RAN, SNUPN, XPO5 }\end{array}$ & 0.000933778 \\
\hline & Pyrimidine metabolism & 2 & POLE3, CMPK1 & 0.034919775 \\
\hline \multirow[t]{4}{*}{ B } & RNA transport & 9 & $\begin{array}{c}\text { EIF3B, EIF3C, EIF3C, EIF4A1, } \\
\text { EIF3H, EIF4B, EIF3F, EIF1B, } \\
\text { EIF4A2 }\end{array}$ & $1.63 \mathrm{E}-05$ \\
\hline & Viral myocarditis & 1 & EIF4G2 & 0.07922721 \\
\hline & Proteoglycans in cancer & 1 & EIF4B & 0.134513214 \\
\hline & mTOR signaling pathway & 1 & EIF4B & 0.255698228 \\
\hline
\end{tabular}

PCCB, propionyl-CoA carboxylase beta subunit; SDHA, succinate dehydrogenase complex flavoprotein subunit A; HADH, hydroxyacyl-CoA dehydrogenase; IDH1, isocitrate dehydrogenase (NADP(+)) 1, cytosolic; NXF1, nuclear RNA export factor 1; NUP, nucleoporin; KPNB1, karyopherin subunit $\beta 1$; XPO, exportin; RAN, RAN member RAS oncogene family; SNUPN, snurportin 1; POLE3, DNA polymerase $\varepsilon$, accessory subunit; cytidine/uridine monophosphate kinase 1; mTOR, mechanistic target of rapamycin; EIF, eukaryotic translation initiation factor.

A

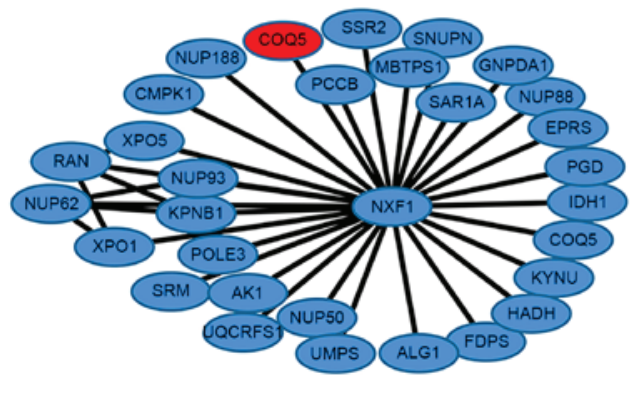

B

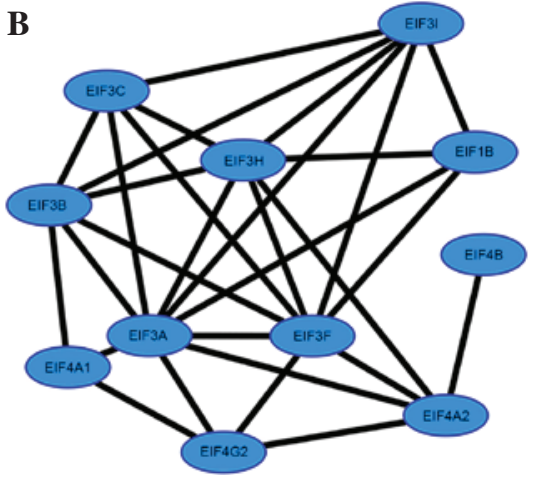

Figure 2. The top two modules (A and B) drawn from the protein-protein interaction network of the differentially expressed genes. Blue genes were downregulated and the red gene was upregulated.

A

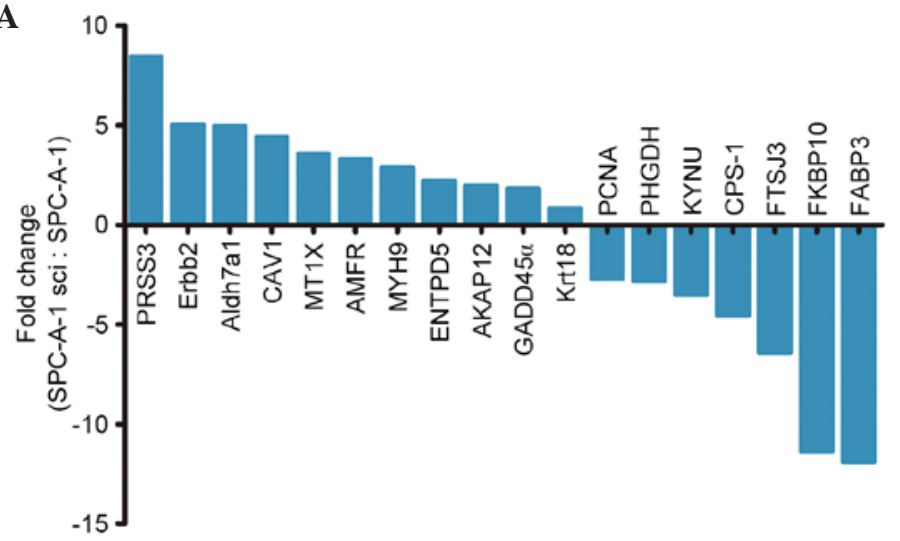

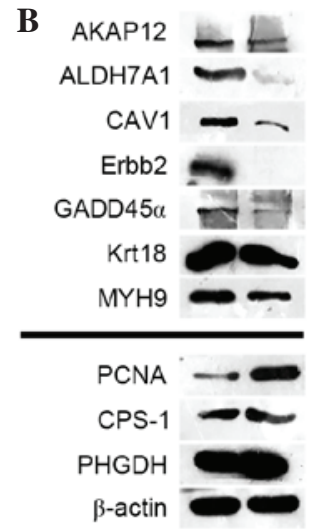

Figure 3. Validation of the expression for DEGs on the protein and mRNA levels. (A) Validation of 18 DEGs at the mRNA level. (B) Validation of 10 DEGs at the protein level. DEGs, differentially expressed genes; PRSS3, protease, serine 3; Erbb2, erb-b2 receptor tyrosine kinase 2; Aldh7a1, aldehyde dehydrogenase 7 family member A1; CAV1, caveolin 1; MT1X, metallothionein 1X; AMFR, autocrine motility factor receptor; MYH9, myosin heavy chain 9; ENTPD5, ectonucleoside triphosphate diphosphohydrolase 5; AKAP12, A-kinase anchoring protein 12; GADD45 $\alpha$, growth arrest and DNA damage inducible $\alpha$; Krt18, keratin 18; PCNA, proliferating cell nuclear antigen; PHGDH, phosphoglycerate dehydrogenase; KYNU, kynureninase; CPS-1, carbamoyl-phosphate synthase 1; FTSJ3, FtsJ homolog 3; FKBP10, FK506 binding protein 10; FABP3, fatty acid binding protein 3. 
A

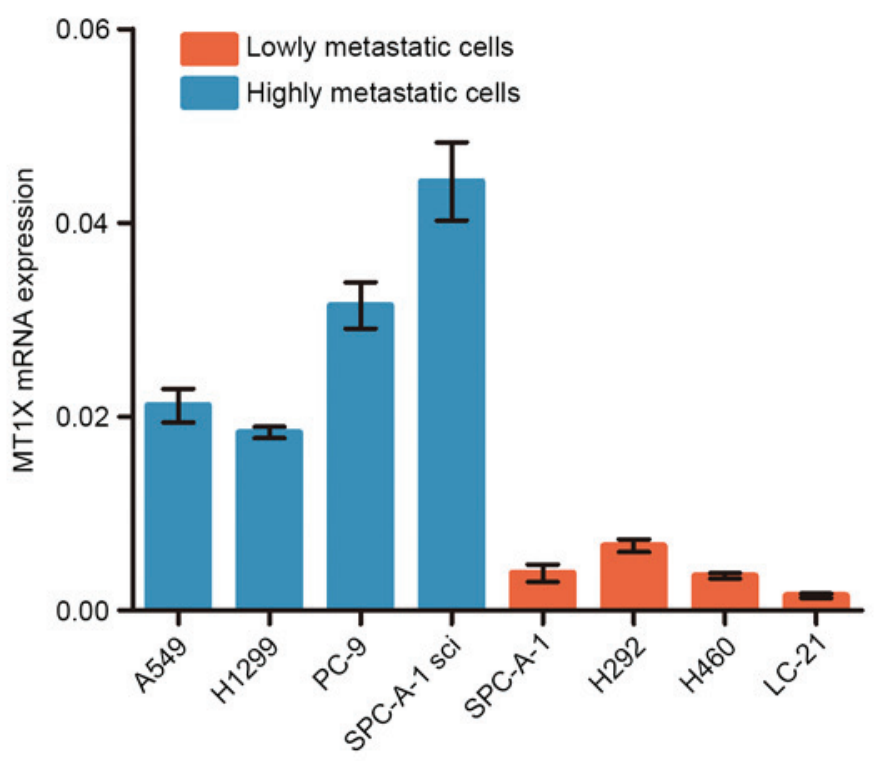

B

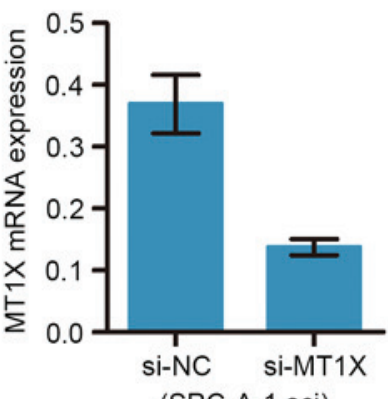

(SPC-A-1 sci)

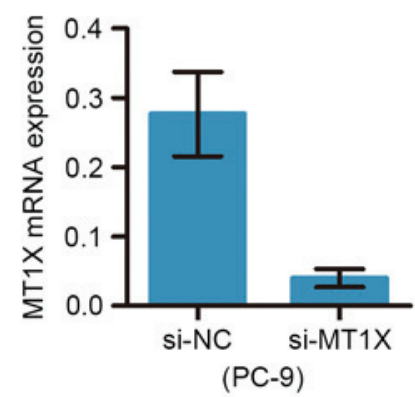

C

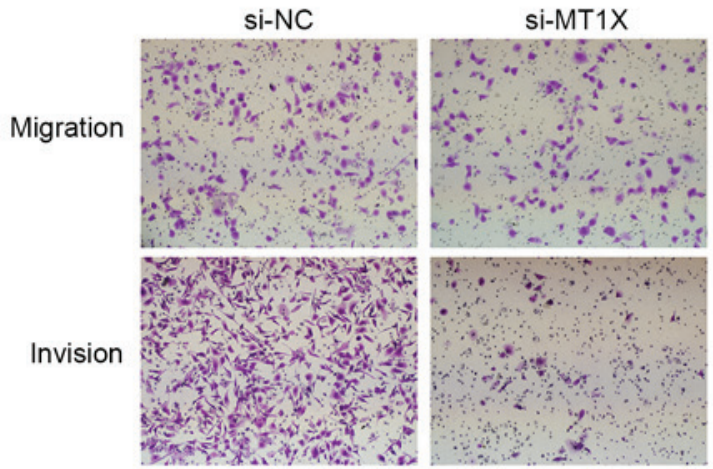

(SPC-A-1 sci)

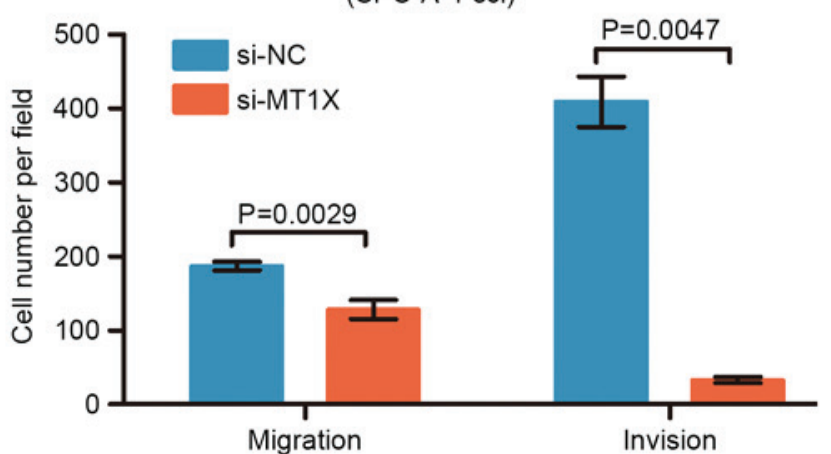

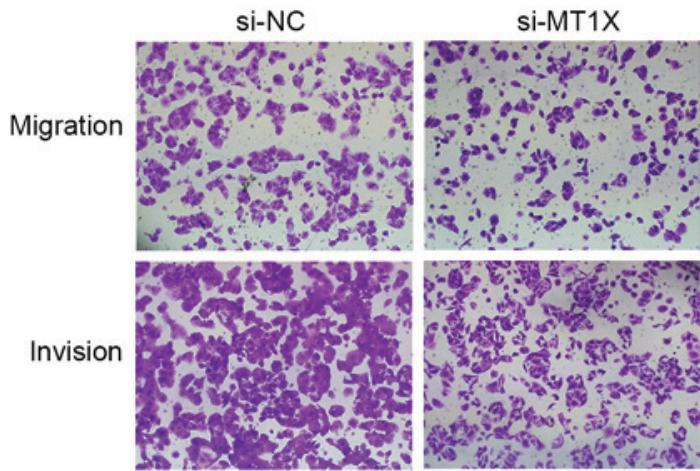

(PC-9)

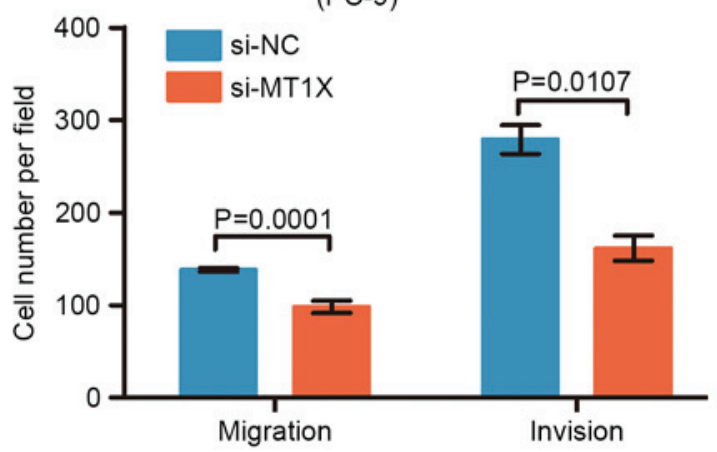

Figure 4. Validation of the expression and function for MT1X in NSCLC cell lines. (A) RT-qPCR analysis to quantify the expression levels of MT1X in NSCLC cells. (B) RT-qPCR analyses of MT1X expression in SPC-A-1sci and PC-9 cells transfected with si-MT1X. (C) Transwell migration and invasion assays in SPC-A-1sci and PC-9 cells were determined following transfection with si-MT1X. NSCLC, non-small cell lung cancer; RT-qPCR, reverse transcription-quantitative polymerase chain reaction; MT1X, metallothionein 1X; si, small interfering RNA; NC, negative control.

in the metastasis of NSCLC (27-33). NME/NM23 nucleoside diphosphate kinase 1 (NME1) was identified to be involved in migration and invasion in numerous other types of cancer (34). According to the literature searching and the microarray results, the dysregulated genes were separated into three classes: Genes that had been reported only in tumor; genes that had been reported in tumors and in tumor metastasis; and genes that had been reported in neither. Due to the large number of dysregulated genes, every gene could not be validated. Therefore, 18
DEGs were selected to further test the validity of the microarray results. Among the 18 genes, protease, 3 (PRSS3), MT1X, CAV1, Erbb2, GADD45 $\alpha$, Krt18, MYH9 and PCNA have been previously reported to be involved in tumors and in tumor metastasis. CPS1, autocrine motility factor receptor (AMFR), ectonucleoside triphosphate diphosphohydrolase 5 (ENTPD5), AKAP12, ALDH7A1, PHGDH, FK506 binding protein 10 (FKBP10) and fatty acid binding protein 3 (FABP3) have been previously reported to be involved in tumors, however, not in 
A

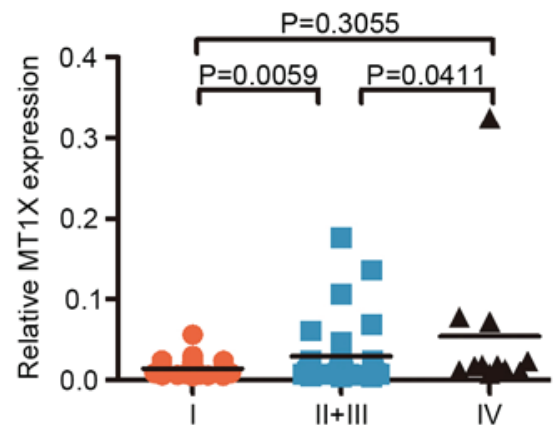

B

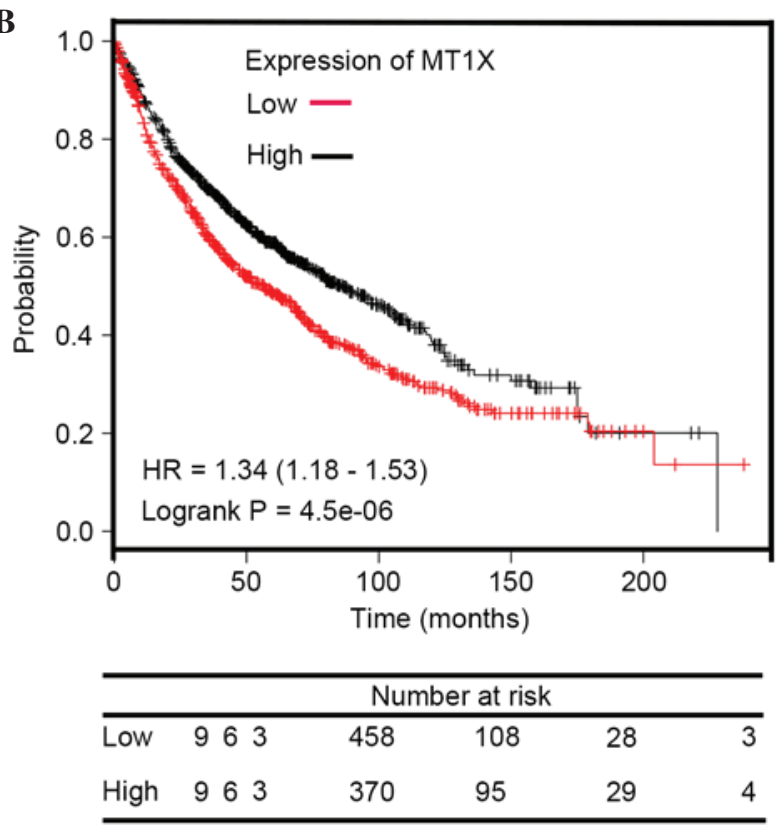

Figure 5. Analysis of the clinical value of MT1X. (A) Expression of MT1X is correlated with advanced clinical stage and is measured using reverse transcription-quantitative polymerase chain reaction in 72 pairs of non-small cell lung cancer samples and corresponding non-cancerous samples. (B) Survival analysis for 1928 cases of lung cancer patients to discover the association between poor prognosis and MT1X with Kaplan Meier-plotter. MT1X, metallothionein $1 \mathrm{X}$; HR, hazard ratio.

tumor metastasis. kynureninase (KYNU) and FtsJ homolog 3 (FTSJ3) have not been previously reported in tumors or in tumor metastasis. The mRNA levels of the 11 upregulated (PRSS3, MT1X, AMFR, ENTPD5, AKAP12, ALDH7A1, CAV1, ERBB2, GADD45 $\alpha$, Krt18 and MYH9) and 7 downregulated genes (PHGDH, KYNU, FTSJ3, FABP3, CPS1, PCNA and FKBP10) were verified in the SPC-A-1sci and SPC-A-1 cell lines with RT-qPCR. The expression level of the 11 upregulated genes were higher in SPC-A-1sci compared with the SPC-A-1 cells, and the expression levels of the 7 downregulated genes were significantly lower in the SPC-A-1sci compared with the SPC-A-1 cells (Fig. 3A). Due to the limited antibodies and the large number of candidate proteins (18 proteins), the range of the noteworthy candidates was restricted to 10 proteins (including 7 upregulated proteins, AKAP12, ALDH7A1, CAV1, ERBB2, GADD45A, Krt18 and MYH9, and 3 downregulated proteins, PHGDH, PCNA and CPS1) to verify the expression on the protein levels in the SPC-A-1sci and SPC-A-1 cell lines. The mRNA and protein levels were validated as consistent with the microarray results (Fig. 3B).
SPC-A-1sci cells transfected with siRNA against MTIX exhibit low migration and invasion abilities in vitro. The accuracy of the microarray can be verified with further experiments. Therefore, the present study attempted to identify MRGs for NSCLC by combining array analysis with in vitro functional assays. Depending on the previous expression verification and literature searching, 3 upregulated genes, MT1X, AMFR and ENTPD5, were selected for further study. According to previous studies, A549, H1299, PC-9 and SPC-A-1sci were revealed to exhibit higher metastasis ability compared with LC-21, SPC-A-1, H460 and H292 (35,36). Following analysis of the 3 noteworthy genes, only MT1X expression levels in these cell lines were demonstrated to be markedly overexpressed in the highly metastatic cell lines (A549, H1299, PC-9 and SPC-A-1sci) compared with the weakly metastatic cells (LC-21, SPC-A-1, H358 and H292) (Fig. 4A). Therefore, the present study focused on exploring the function of MT1X in the metastasis of lung cancer. The SPC-A-1sci and PC-9 cells were transfected with MT1X siRNA and migration and invasion assays were performed. Effective knockdown of MT1X in the two NSCLC cell lines was confirmed with RT-qPCR (Fig. 4B). Notably, when compared with the control group, the cells transfected with si-MT1X exhibited significantly lower migration and invasion abilities compared with negative control cells in SPC-A-1sci and PC-9 (Fig. 4C). The results indicate a potential role of MT1X in lung cancer metastasis.

MT1X expression levels in lung cancer are associated with advanced clinical stage and indicate a poor prognosis. To further validate the function of MT1X in lung cancer, its expression level was compared in human lung cancer tissues $(\mathrm{n}=72)$ and matched adjacent normal lung tissues using RT-qPCR. The stage for patients was classed in accordance with the 7th Edition of the AJCC Cancer Tumor Node Metastasis Classification (37). A prominent statistical difference between stages I and IV was revealed following the collection of all the data (Fig. 5A). Therefore, the upregulation of MT1X appeared to be associated with a higher clinical stage. Kaplan Meier-plotter (www.kmplot.com) is highly valuable for researchers making the preliminary assessment of biomarkers. In 2009, Györffy el al (38) integrated a database with a Kaplan Meier-plotter to detect the prognostic value of new biomarkers and search biomarker candidates in NSCLC. In 2010, Győrffy et al (39) used the Kaplan Meier-plotter to perform survival analysis and confirm the prognostic power of the proliferation-related genes. In the present study, an integrative data analysis was performed with the Kaplan Meier-plotter and the results suggested that a high expression level of MT1X indicated a poor prognosis in lung cancer, as well as in breast cancer (Fig. 5B). Overall, with respect to the clinical features, the high expression of MT1X was positively correlated with clinical stage, indicating its important role in the metastatic process of NSCLC and poor prognosis in patients.

\section{Discussion}

mRNA expression profiles were conducted in SPC-A-1sci and SPC-A-1 cell lines to detect the MRGs. A total of 4,838 DEGs were screened, including 798 upregulated and 4,040 downregulated genes. Systematic analysis was performed to 
establish the validity of the microarray, and identify the possible MRGs. Firstly, a comprehensive bioinformatics analysis of DEGs was performed. With the KEGG database, DEGs were revealed to be predominantly enriched in the biosynthesis of antibiotics, carbon metabolism, spliceosome and ribosome categories. Furthermore, DEGs were identified by the BioGRID 3.4 database and visualized by Cytoscape 3.2 to form an integral PPI network. Modular analysis of the PPI network was performed with ClusterONE and the two main modules were selected for further analysis. In module A, 31 DEGs were enriched in the biosynthesis of antibiotics, protein processing in endoplasmic reticulum, RNA transport and pyrimidine metabolism. The genes were mostly centered in the RNA transport pathway. Its 'hub' gene NXF1, with a degree of 107, was reported to be a potential therapeutic target in cancer. In module B, DEGs were predominantly enriched in RNA transport, viral myocarditis, proteoglycans in cancer and the mTOR signaling pathway. Module B consisted of 11 genes and all of its 'hub' genes (EIF3A, EIF3F and EIF3B) were extensively reported to have an association with cancer. The EIF family and NXF1 are predominantly enriched in the RNA transport pathway. RNA transport has a correlation with cell migration and tumor cell metastasis, therefore, we can possibly regard the DEGs enriched in RNA transport and establish their potential function in NSCLC (40). When scanning the 4,838 DEGs, EGFR, FGFR3, S100P, S100A2, MTA1 and mTOR were widely recognized as involved in the metastasis of NSCLC, and Erbb2 and NME1 were identified as genes that were associated with migration and invasion in numerous other types of cancer. The aforementioned findings verified that the present microarray results contain a number of tumor-associated genes. For further confirmation of the microarray, 4,838 DEGs were classed into three groups. A combined literature search with this classification, allowed the selection of 18 genes to detect the expression of the mRNA level, and 10 of these 18 genes detect the expression at the protein level. All the test results were consistent with the microarray.

In confirming the accuracy of the microarray, more confidence was observed when selecting the novel MRGs for NSCLC from the microarray. Three upregulated genes (MT1X, AMFR and ENTPD5) were selected for further study and the difference in their expression levels was detected in four highly (A549, H1299, PC-9 and SPC-A-1sci) and four weakly metastatic NSCLC cell lines (LC-21, SPC-A-1, H460 and H292). The MT1X expression levels were markedly overexpressed in highly metastatic compared with weakly metastatic cells. Following transfection with siRNA-MT1X in the SPC-A-1sci and PC-9 cell lines, the two transfected cell lines exhibited significantly lower migration and invasion abilities compared with the control cells. Furthermore, the expression of MT1X in 72 pairs of clinical samples revealed a prominent statistical difference in expression between stages I and IV. The association between a poor prognosis and MT1X expression in lung cancer was demonstrated by a Kaplan Meier-plotter with a total of 1,928 cases of patients with lung cancer. The aforementioned results suggested that MT1X is associated with the metastatic ability in NSCLC and indicates a poor prognosis in patients with lung cancer. Therefore, MT1X is a potential prognostic clinical marker for NSCLC.
However, further investigations are required to elucidate the metastatic-promoting mechanisms of MT1X.

In conclusion, the present study identified DEGs using bioinformatics analysis and in vivo assays, and proved that this mRNA microarray has clinical value in screening genes involved in metastasis in NSCLC. Future experiments are required to confirm the role and reveal the functional mechanism of MT1X. The detection of additional MRGs from the microarray is required in order to explore the molecular mechanisms of NSCLC metastasis.

\section{References}

1. Torre LA, Bray F, Siegel RL, Ferlay J, Lortet-Tieulent J and Jemal A: Global cancer statistics, 2012. Cancer J Clin 65: 87-108, 2015.

2. Siegel RL, Miller KD and Jemal A: Cancer statistics, 2015. CA Cancer J Clin 65: 5-29, 2015.

3. Molina JR, Yang P, Cassivi SD, Schild SE and Adjei AA: Non-small cell lung cancer: Epidemiology, risk factors, treatment, and survivorship. Mayo Clin Proc 83: 584-594, 2008.

4. Gupta GP and Massagué J: Cancer metastasis: Building a framework. Cell 127: 679-695, 2006.

5. Sun L, Li J and Yan B: Gene expression profiling analysis of osteosarcoma cell lines. Mol Med Rep 12: 4266-4272, 2015.

6. Huang HL, Wu YC, Su LJ, Huang YJ, Charoenkwan P, Chen WL, Lee HC, Chu WC and Ho SY: Discovery of prognostic biomarkers for predicting lung cancer metastasis using microarray and survival data. BMC Bioinformatics 16: 54, 2015.

7. Jia DS, Yan M, Wang X, Hao X, Liang L, Liu L, Kong H, He X, $\mathrm{Li} \mathrm{J}$ and Yao M: Development of a highly metastatic model that reveals a crucial role of fibronectin in lung cancer cell migration and invasion. BMC Cancer 10: 364, 2010.

8. Morandi L, de Biase D, Visani M, Monzoni A, Tosi A, Brulatti M, Turchetti D, Baccarini P, Tallini G and Pession A: T([20]) repeat in the 3'-untranslated region of the MT1X gene: A marker with high sensitivity and specificity to detect microsatellite instability in colorectal cancer. Int J Colorectal Dis 27: 647-656, 2012.

9. Peng B, Gu Y, Xiong Y, Zheng G and He Z: Microarray-assisted pathway analysis identifies MT1X \& NFKB as mediators of TCRP1-associated resistance to cisplatin in oral squamous cell carcinoma. PLoS One 7: e51413, 2012.

10. Huang da W, Sherman BT and Lempicki RA: Systematic and integrative analysis of large gene lists using DAVID bioinfor-matics resources. Nat Protoc 4: 44-57, 2009.

11. Harris MA, Clark J, Ireland A, Lomax J, Ashburner M, Foulger R, Eilbeck K, Lewis S, Marshall B, Mungall C, et al: The gene ontology (GO) database and informatics resource. Nucleic Acids Res 32: (Database issue); D258-D261, 2004.

12. Livak KJ and Schmittgen TD: Analysis of relative gene expression data using real-time quantitative PCR and the 2(-Delta Delta C(T)) method. Methods 25: 402-408, 2001.

13. Siddiqui $N$ and Borden KL: mRNA export and cancer. Wiley Interdiscip Rev RNA 3: 13-25, 2012.

14. Hajingabo LJ, Daakour S, Martin M, Grausenburger R, Panzer-Grümayer R, Dequiedt F, Simonis N and Twizere JC: Predicting interactome network perturbations in human cancer: Application to gene fusions in acute lymphoblastic leukemia. Mol Biol Cell 25: 3973-3985, 2014.

15. Zhang Y, Yu JJ, Tian Y, Li ZZ, Zhang CY, Zhang SF, Cao LQ, Zhang Y, Qian CY, Zhang W, et al: eIF3a improve cisplatin sensitivity in ovarian cancer by regulating XPC and p27Kip1 translation. Oncotarget 6: 25441-25451, 2015.

16. Spilka R, Ernst C, Bergler H, Rainer J, Flechsig S, Vogetseder A, Lederer E, Benesch M, Brunner A, Geley S, et al: eIF3a is over-expressed in urinary bladder cancer and influences its phenotype independent of translation initiation. Cell Oncol (Dordr) 37: 253-267, 2014.

17. Shen J, Yin JY, Li XP, Liu ZQ, Wang Y, Chen J, Qu J, Xu XJ, McLeod HL, He YJ, et al: The prognostic value of altered eIF3a and its association with p27 in non-small cell lung cancers. PLoS One 9: e96008, 2014.

18. Marchione R, Laurin D, Liguori L, Leibovitch MP, Leibovitch SA and Lenormand JL: MD11-mediated delivery of recombinant eIF3f induces melanoma and colorectal carcinoma cell death. Mol Ther Methods Clin Dev 2: 14056, 2015. 
19. Wen F, Zhou R, Shen A, Choi A, Uribe D and Shi J: The tumor suppressive role of eIF3f and its function in translation inhibition and rRNA degradation. PLoS One 7: e34194, 2012.

20. Wang H, Ru Y, Sanchez-Carbayo M, Wang X, Kieft JS and Theodorescu D: Translation initiation factor eIF3b expression in human cancer and its role in tumor growth and lung colonization. Clin Cancer Res 19: 2850-2860. 2013.

21. Do SI, Kim YW, Park HR and Park YK: Expression of insulin-like growth factor-II mRNA binding protein 3 (IMP3) in osteosarcoma. Oncol Res 17: 269-272, 2008.

22. Jiang Z, Lohse CM, Chu PG, Wu CL, Woda BA, Rock KL and Kwon ED: Oncofetal protein IMP3: A novel molecular marker that predicts metastasis of papillary and chromophobe renal cell carcinomas. Cancer 112: 2676-2682, 2008

23. Miyamoto T, Asaka R, Suzuki A, Takatsu A, Kashima H and Shiozawa T: Immunohistochemical detection of a specific receptor for lipocalin2 (solute carrier family 22 member 17 SLC22A17) and its prognostic significance in endometrial carcinoma. Exp Mol Pathol 91: 563-568, 2011.

24. Aharinejad S, Salama M, Paulus P, Zins K, Berger A and Singer CF: Elevated CSF1 serum concentration predicts poor overall survival in women with early breast cancer. Endocr Relat Cancer 20: 777-783, 2013.

25. Liu X, Ma D, Jing X, Wang B, Yang W and Qiu W: Overexpression of ANXA2 predicts adverse outcomes of patients with malignant tumors: A systematic review and meta-analysis. Med Oncol 32: 392, 2015.

26. Wan J, Che Y, Kang N and Wu W: SOCS3 blocks HIF-10 expression to inhibit proliferation and angiogenesis of human small cell lung cancer by downregulating activation of Akt, but not STAT3. Mol Med Rep 12: 83-92, 2015.

27. Chien MH, Lee WJ, Hsieh FK, Li CF, Cheng TY, Wang MY, Chen JS, Chow JM, Jan YH, Hsiao M, et al: Keap1-Nrf2 interaction suppresses cell motility in lung adenocarcinomas by targeting the S100P protein. Clin Cancer Res 21: 4719-4732, 2015.

28. Lococo F, Paci M, Rapicetta C, Rossi T, Sancisi V, Braglia L, Cavuto S, Bisagni A, Bongarzone I, Noonan DM, et al: Preliminary evidence on the diagnostic and molecular role of circulating soluble EGFR in non-small cell lung cancer. Int J Mol Sci 16: 19612-19630, 2015.

29. Bulk E, Sargin B, Krug U, Hascher A, Jun Y, Knop M, Kerkhoff C, Gerke V, Liersch R, Mesters RM, et al: S100A2 induces metastasis in non-small cell lung cancer. Clin Cancer Res 15: 22-29, 2009.
30. Li S, Tian H, Yue W, Li L, Gao C, Si L, Li W, Hu W, Qi L and $\mathrm{Lu}$ M: Down-regulation of MTA1 protein leads to the inhibition of migration, invasion, and angiogenesis of non-small-cell lung cancer cell line. Acta Biochim Biophys Sin (Shanghai) 45: 115-122, 2013.

31. Zhu X, Zhang X, Wang H, Song Q, Zhang G, Yang L, Geng J, Li X, Yuan Y and Chen L: MTA1 gene silencing inhibits invasion and alters the microRNA expression profile of human lung cancer cells. Oncol Rep 28: 218-224, 2012.

32. Li L, Liu D, Qiu ZX, Zhao S, Zhang L and Li WM: The prognostic role of mTOR and p-mTOR for survival in non-small cell lung cancer: A systematic review and meta-analysis. PLoS One 10: e0116771, 2015.

33. Liu J, Cho SN, Akkanti B, Jin N, Mao J, Long W, Chen T, Zhang Y, Tang X, Wistub II, et al: ErbB2 pathway activation upon Smad4 Loss promotes lung tumor growth and metastasis. Cell Rep pii: S2211-S1247, 2015.

34. McCorkle JR, Leonard MK, Kraner SD, Blalock EM, Ma D, Zimmer SG and Kaetzel DM: The metastasis suppressor NME1 regulates expression of genes linked to metastasis and patient outcome in melanoma and breast carcinoma. Cancer Genomics Proteomics 11: 175-194, 2014.

35. Yu T, Li J, Yan M, Liu L, Lin H, Zhao F, Sun L, Zhang Y, Cui Y, Zhang F, et al: MicroRNA-193a-3p and -5p suppress the metastasis of human non-small-cell lung cancer by downregulating the ERBB4/PIK3R3/mTOR/S6K2 signaling pathway. Oncogene 34: 413-423, 2015.

36. Yu T, Liu L, Li J, Yan M, Lin H, Liu Y, Chu D, Tu H, Gu A and Yao M: miRNA-10a is upregulated in NSCLC and may promote cancer by targeting PTEN. Oncotarget 6: 30239-30250, 2015.

37. Talsma K, van Hagen P, Grotenhuis BA, Steyerberg EW, Tilanus HW, van Lanschot JJ and Wijnhoven BP: Comparison of the 6th and 7th Editions of the UICC-AJCC TNM Classification for Esophageal Cancer. Annals of Surgical Oncology. July 2012, Volume 19, Issue 7, 2142-2148.

38. Györffy B, Lanczky A, Eklund AC, Denkert C, Budczies J, Li Q and Szallasi Z: An online survival analysis tool to rapidly assess the effect of 22,277 genes on breast cancer prognosis using microarray data of 1,809 patients. Breast Cancer Res Treat 123: 725-731, 2010.

39. Győrffy B, Surowiak P, Budczies J and Lánczky A: Online survival analysis software to assess the prognostic value of biomarkers using transcriptomic data in non-small-cell lung cancer. PLoS One 8: e82241, 2013.

40. Shankar J and Nabi IR: RNA purification from tumor cell protrusions using porous polycarbonate filters. Methods Mol Biol 714: 353-366, 2011. 